\title{
The safety and accuracy of ECG-guided PICC tip position verification applied in patients with atrial fibrillation
}

This article was published in the following Dove Press journal: Therapeutics and Clinical Risk Management

\author{
Yufang Gao',* \\ Yuxiu Liu ${ }^{1,2, *}$ \\ Hui Zhangl,* \\ Fang Fang ${ }^{3}$ \\ Lei Song ${ }^{4}$
}

'Hospital Management Office, The Affiliated Hospital of Qingdao University, Qingdao, People's Republic of China; ${ }^{2}$ Department of Community Nursing, School of Nursing, Weifang Medical University, Weifang, People's Republic of China; ${ }^{3}$ Department of Hematology, The Affiliated Hospital of Qingdao University, Qingdao, People's Republic of China; ${ }^{4}$ Intensive Care Unit, The Affiliated Hospital of Qingdao University, Qingdao, People's Republic of China

*These authors contributed equally to this work
Correspondence: Yufang Gao

Hospital Management Office,

The Affiliated Hospital of Qingdao

University, No 16 Jiangsu Road, Shinan

District, Qingdao, 266005, People's

Republic of China

Tel +86 I86 6I80 I800

Email gaoyufang06I2@I63.com
Background: Tip position verification of peripherally inserted central catheters (PICCs) is essential to the use of the catheter. Postprocedural chest X-ray as the "gold standard" practice for PICC tip confirmation can lead to a significant delay for patient IV therapy, cost more, and lead to radiation exposure for both patients and staffs. Intracavitary electrocardiogram (IC-ECG)-guided PICC placement which provides real-time tip confirmation during the insertion procedure has been widely used. However, safety and accuracy of ECG for abnormal surface ECG patients, such as patients with atrial fibrillation (AF), have not been reported.

Objective: To determine the safety and accuracy of IC-ECG technique for PICC tip position verification among the patients with AF.

Patients and methods: A prospective cohort study was conducted in a teaching and tertiary referral hospital with more than 3,600 beds in Qingdao, People's Republic of China. Adult patients with diagnosis of AF who need a PICC for infusion from June 2015 to May 2017 were enrolled in the study. For every included patient with AF, ECG was used to detect the PICC tip position during catheterization and X-ray was done to confirm the tip position as the "gold standard" after PICC insertion. The effectiveness and accuracy of ECG-guided catheter tip positioning and chest X-ray confirmation were compared.

Results: Totally, 118 AF patients with 118 PICCs were enrolled (58 male and 60 female, age range 50-89 years old). There was no catheterization-related complication. When the catheter entered the lower $1 / 3$ of superior vena cava, the amplitude of $f$ wave reached the maximum. There was no statistical difference between X-ray PICC tip position verification and IC-ECG PICC tip position verification among patients with $\mathrm{AF}\left(\chi^{2}=1.31, P=0.232\right)$. Utilizing the cutoff point of f wave change $\geq 0.5 \mathrm{~cm}$, a sensitivity of 0.94 , a specificity of 0.71 , a positive predictive value of 0.98 , and a negative predictive value of 0.42 were observed. The area under the receiver operating characteristic curve was 0.909 (95\% CI: 0.810-1.000).

Conclusion: The ECG-guided technique represents a safe and accurate technique to verify the position of PICC tip in patients with AF and could potentially remove the requirement for postprocedural chest X-ray among the patients with AF.

Keywords: peripherally inserted central catheter, PICC, tip position, electrocardiograph, ECG, patients with atrial fibrillation

\section{Introduction}

Correct tip positioning of peripherally inserted central catheters (PICCs) is crucial to avoid catheterization-related complications such as migration, venous thrombosis, or arrhythmia. ${ }^{1}$ There are several methods for PICC tip positioning, postprocedural chest X-ray, electrocardiogram (ECG), and some reported novel techniques such 
as Sherlock $3 \mathrm{CG}^{\circledR}$ Tip Confirmation System (Bard Access Systems, Inc., Salt Lake City, UT, USA) which integrated magnetic tracking and ECG-based PICC tip confirmation technology ${ }^{2}$ and conductance guidewire system. ${ }^{3}$

The chest X-ray is the most frequently used method to verify PICC tip position and was recommended as the gold standard. ${ }^{4}$ One of the limitations of X-ray is that the postprocedural confirmation may lead to a significant delay in a patient intravenous (IV) therapy. ${ }^{5}$ Moreover, if incorrect PICC tip position is detected by postprocedural X-ray, repeated catheter manipulation and chest X-ray are required, which leads to a delay in patient treatment and further time use with increased cost. In addition, there is a possibility for complications, including catheter-related bloodstream infection owing to the integrity of the dressing being interrupted. ${ }^{6,7}$ The additional time, cost, and radiation exposure involved with radiographic assessment lead to PICC placement only within a hospital setting. ${ }^{1}$

ECG technique used in central venous catheter (CVC) tip positioning was first reported in $1949 .{ }^{8}$ Intracavitary ECGguided PICC placement provides real-time tip confirmation during the insertion procedure. More and more evidence indicated ECG-guided PICC tip positioning could be as accurate as X-ray method. ${ }^{5,9-11}$ Walker's systematic review suggested ECG-based positioning could potentially remove the requirement for postprocedural chest X-ray, especially during PICC line insertion. ${ }^{6}$ ECG-guided PICC tip location is clarified live during catheter insertion with no need for postprocedural adjustment or repositioning. The PICC can be used straight after placement with no time delay for patient treatment. ${ }^{9}$

The current standard for PICC tip position is the lower one-third of the superior vena cava (SVC) at the caval-atrial junction (CAJ). ${ }^{10,11}$ As the PICC tip approaches the sinoatrial node at $\mathrm{CAJ}$, the $\mathrm{P}$ wave starts to elevate, reaching its maximum amplitude at the CAJ. When it passes through into the right atrium, the $\mathrm{P}$ wave starts to invert, indicating the PICC is inserted too far. The ideal PICC tip position is where the ECG shows maximal P amplitude. Since ECG-guided PICC tip positioning is regarded as a safe, reliable, and replication method, can it be used in patients with atrial fibrillation (AF) who have no P wave in their ECG? Our research team is from the Affiliated Hospital of Qingdao University where about 5,000 PICCs are inserted annually. The research team has been working on the research of PICCs, and during our research, we found that $\mathrm{AF}$ patients also had some distinct changes in the f waves during the PICC tip positioning. The team consequently explored this.

\section{Patients and methods}

\section{Patients}

This prospective cohort study was conducted in the Affiliated Hospital of Qingdao University, a tertiary referral hospital with more than 3,600 beds from June 2015 to May 2017. The study protocol was reviewed and approved by institutional review board of the Affiliated Hospital of Qingdao University (approval no. QDFYLL201422). All the patients enrolled signed written informed consent.

The inclusion criteria consisted of the following: 1) patients needing a PICC whose ECG showed AF wave before PICC insertion; 2) over 18 years old; 3) patients tolerable to X-ray examination. Exclusion criteria were as follows: 1) patients suffering from mental illness or skin diseases; 2) patients with a heart pacemaker; 3 ) patients with any other types of catheter 4) patients allergic to alcohol and iodophor.

\section{PICC insertion procedure}

PICC was inserted by professional PICC nurses with ultrasound guidance under standard sterile conditions. Four French (Fr) single-lumen distal valved silicone Bard Groshong ${ }^{\circledR}$ PICC (Bard Access Systems, Inc.) was used in the study. Bard Site Rite 5 Sonogram Machine Ultrasound System (Bard Access Systems, Inc.) was used to measure and evaluated relevant veins at the insertion point. All PICCs were inserted by the modified Seldinger technique using Groshong ${ }^{\circledR}$ NXT ClearVue. All the PICCs were flushed with $10 \mathrm{~mL}$ saline after catheterization and the catheter entrance sites were covered with dressing after skin disinfection. Chest radiography was routinely examined to confirm the catheter tip position. ${ }^{10}$

\section{Tip confirmation by X-ray}

According to the Infusion Nurses Society, the recommended tip position is located at the lower third of the SVC close to the entrance to the right atrium. ${ }^{11}$ It was reported that CVC tip position approximately $4 \mathrm{~cm}(95 \% \mathrm{CI}$ : $3.8-4.3 \mathrm{~cm}$ ) below the carina would result in placement near the CAJ and the average length of SVC was $7.1 \mathrm{~cm} .{ }^{12}$ In this study, we used X-ray method as the "gold standard" to confirm PICC tip position. When taking X-ray examination, all patients took a neutral supine position, arms along the body, and with no forced breathing to avoid possible tip malposition by position or forced inspiration. We utilized carina as an anatomical landmark from which to measure the PICC tip. The optimal position was estimated to be 1.6-4 cm under the carina in our study. ${ }^{12,13}$ The X-ray data were evaluated 
separately by 2 radiologists. If there was inconsistent judgment, a third radiologist would further check the X-ray result and confirm a decision.

\section{Intraprocedural verification of tip position by ECG method}

The ECG in the study was obtained by so-called "saline technique" which uses a column of saline solution contained in the catheter as an intracavitary electrode. ${ }^{13}$ Braun ${ }^{\circledR}$ transducer and switch for shifting from surface ECG tracing to intracavitary ECG (IC-ECG) tracing were used in the study. Three surface electrodes (right arm [RA], left arm, and left leg) and lead II were connected. When the catheter tip entered SVC, the catheter was attached to the connector of the transducer, and then a continuous infusion of saline solution through PICC was performed. The ECG in patients with AF shows f wave instead of $\mathrm{P}$ wave. As the catheter tip goes deeper, f wave also undergoes certain changes. When the catheter proceeds into SVC, the f wave gets higher which is similar to $\mathrm{P}$ wave change, that is, when the catheter gets into the SVC, the amplitude of $\mathrm{f}$ wave gradually increases. When the catheter enters the lower $1 / 3$ of SVC, the amplitude of the $f$ wave reaches the maximum, and when the catheter enters the right atrium, the amplitude of the f wave falls.

\section{Efficacy evaluation and data collection}

For every PICC insertion, the following data were collected: 1) patient's data, containing age, sex, diagnosis, and CVC history; 2) PICC insertion data, containing PICC insertion aim, side of insertion arm, vein of puncture, and possible puncture-related complication; 3) intracavitary ECG data, containing the surface ECG amplitude before PICC insertion, peak of f wave while entering the lower $1 / 3$ of SVC, and possible intraoperative complication related to IC-ECG; 4) X-ray data on PICC tip position. All the data were collected by the authors and all PICCs were inserted by PICC specialty nurses with at least 5-year experience in PICC placement. ECG was confirmed by a cardiologist and the authors.

\section{Statistical analysis}

The data were recorded and analyzed using SPSS 19.0 (IBM Corporation, Armonk, NY, USA). Continuous variables were presented as mean $\pm \mathrm{SD}$. $\chi^{2}$ test was used to evaluate the difference of enumeration data. The sensitivity and specificity of $\mathrm{f}$ amplitude in 1/3 SVC compared with X-ray as "gold standard" were calculated. The significance level was set at 0.05 .

\section{Results Study population}

From June 2015 to May 2017, 118 AF patients with 118 PICCs who met the eligibility criteria were enrolled in the study and retained for analysis. There were 58 men (49.15\%) and 60 women $(50.85 \%)$, with a mean age of $67.23 \pm 10.12$ years (range, 50-89 years old). Of the 118 patients with AF, 16 patients were inserted PICCs for parenteral nutrition, 90 for chemotherapy, and 12 with poor peripheral vein condition for long-term infusion. There was no catheterizationrelated complication. The baseline characteristics of the 118 PICCs are listed in Table 1.

\section{Change of $f$ wave amplitude as the tip entered lower I/3 of SVC}

In this study, we found that the f wave amplitude changed as the PICC tip went deeper at SVC. When the catheter entered into SVC, f wave got higher, which was similar to $\mathrm{P}$ wave change of the normal surface ECG patients. When the catheter entered the lower $1 / 3$ of SVC, the amplitude of

Table I Baseline demographics, PICC insertions, and clinical characteristics

\begin{tabular}{|c|c|c|c|c|c|}
\hline \multirow[t]{2}{*}{ Variables } & \multirow[t]{2}{*}{$\mathbf{N}(\%)$} & \multicolumn{2}{|c|}{$\begin{array}{l}\text { IC-ECG f } \\
\text { amplitude } \\
\geq 0.5 \mathrm{~cm} \\
\text { higher } \mathrm{N}\end{array}$} & \multirow[t]{2}{*}{$\chi^{2}$} & \multirow[t]{2}{*}{$P$-values } \\
\hline & & Yes & No & & \\
\hline Sex & & & & 1.338 & 0.247 \\
\hline Male & $58(49.2)$ & 54 & 4 & & \\
\hline Female & $60(50.8)$ & 52 & 8 & & \\
\hline Diagnosis & & & & 0.667 & 0.955 \\
\hline Hematopoietic malignancies & $29(24.6)$ & 25 & 4 & & \\
\hline Gynecologic malignancies & $19(16.1)$ & 17 & 2 & & \\
\hline Breast cancer & $21(17.8)$ & 19 & 2 & & \\
\hline Gastrointestinal malignancies & $27(22.9)$ & 25 & 2 & & \\
\hline Other diseases & $22(18.6)$ & 20 & 2 & & \\
\hline PICC insertion aim & & & & 2.143 & 0.342 \\
\hline Parenteral nutrition & $16(13.6)$ & 13 & 3 & & \\
\hline Chemotherapy & $90(76.3)$ & 83 & 7 & & \\
\hline Long-term infusion & $12(10.2)$ & 10 & 2 & & \\
\hline Side of insertion arm & & & & 0.000 & 1.000 \\
\hline Right upper extremity & $92(78.0)$ & 83 & 9 & & \\
\hline Left upper extremity & $26(22.0)$ & 24 & 2 & & \\
\hline Vein of puncture & & & & 1.790 & 0.409 \\
\hline Basilic vein & 91 (77.1) & 83 & 8 & & \\
\hline Brachial vein & $24(20.3)$ & 20 & 4 & & \\
\hline Cephalic vein & $3(2.5)$ & 3 & 0 & & \\
\hline CVC history & & & & 0.643 & 0.423 \\
\hline Yes & $24(20.3)$ & 20 & 4 & & \\
\hline No & $94(79.7)$ & 86 & 8 & & \\
\hline
\end{tabular}

Abbreviations: IC-ECG, intracavitary electrocardiogram; N, number; PICC, peripherally inserted central catheter; CVC, central venous catheter. 

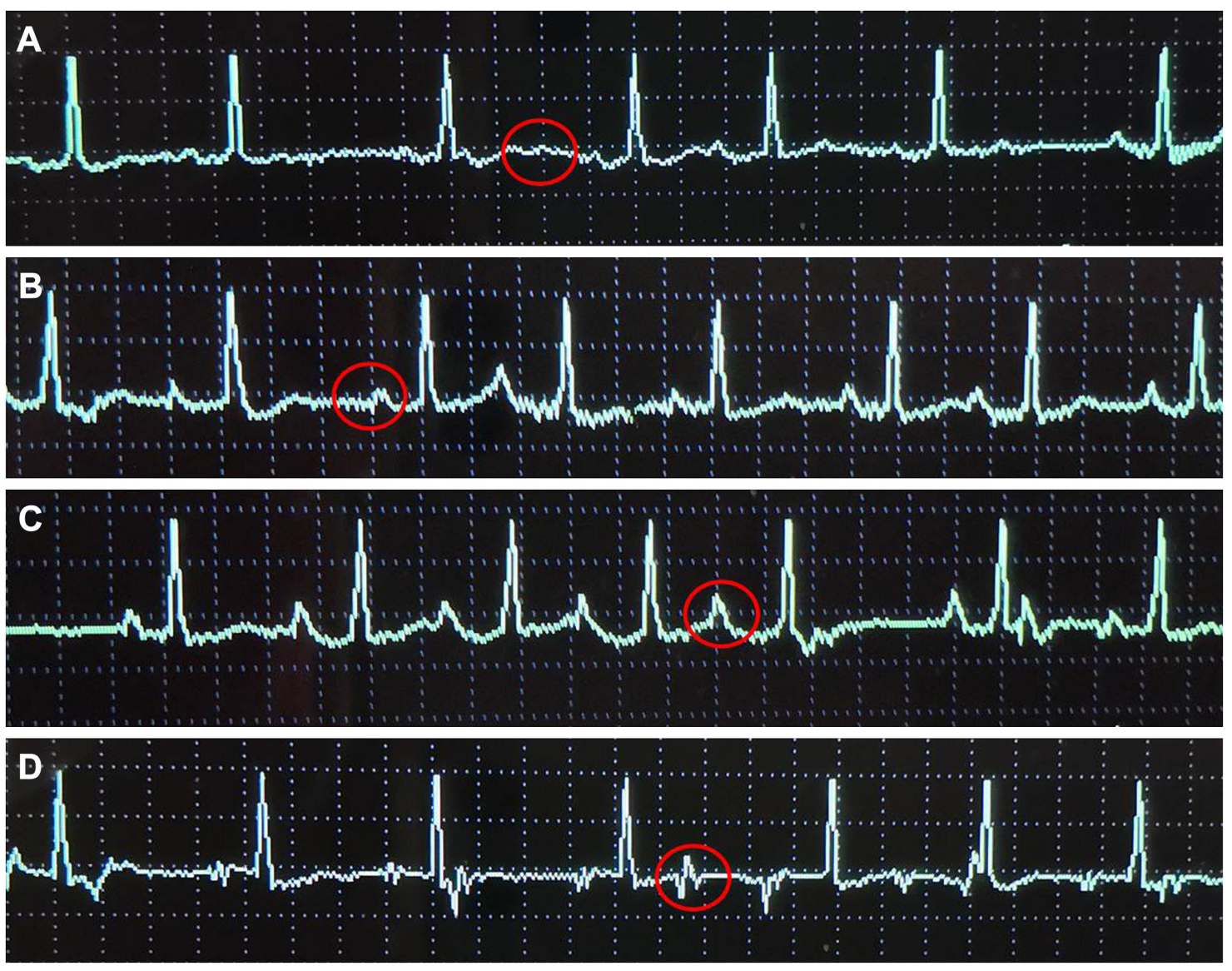

Figure I Change of $f$ wave as PICC entered the lower I/3 of SVC, CAJ, and the right atrium.

Notes: The surface ECG before PICC insertion (A), the ECG when PICC tip approached the lower I/3 of SVC (B), the ECG when PICC tip approached CAJ (C), the ECG when PICC tip entered the right atrium (D). Red circles $=f$ wave.

Abbreviations: CAJ, caval-atrial junction; ECG, electrocardiogram; PICC, peripherally inserted central catheter; SVC, superior vena cava.

f wave reached the maximum. When the catheter entered the right atrium, the amplitude of the $\mathrm{f}$ wave began to fall. Figure 1 shows the surface ECG and the IC-ECG when PICC tip entered the lower $1 / 3$ of SVC in a patient with AF.

When the $\mathrm{f}$ wave reached the maximum amplitude, it was recorded as $\mathrm{f}^{\prime}$. The " $\mathrm{f}$ " stood for the average surface ECG $\mathrm{f}$ amplitude. The average of surface ECG $f$ wave amplitude, $f^{\prime}$, and $\left(f^{\prime}-f\right) / f$ were calculated. The detailed data are listed in Table 2.

Table 2 Average height of $\mathrm{f}$ wave and $95 \% \mathrm{Cl}$

\begin{tabular}{|c|c|c|c|c|}
\hline \multirow[t]{2}{*}{ Variables } & \multirow[t]{2}{*}{$\mathbf{N}$} & \multirow{2}{*}{$\begin{array}{l}\text { Average } \\
\text { height }(\mathrm{cm})\end{array}$} & \multicolumn{2}{|l|}{$95 \% \mathrm{Cl}$} \\
\hline & & & Lower & Upper \\
\hline$f$ & 118 & $1.06 \pm 0.25$ & 1.015 & 1.105 \\
\hline$f^{\prime}$ & 118 & $1.68 \pm 0.34$ & 0.619 & I.74I \\
\hline$\left(f^{\prime}-f\right) / f$ & 118 & $0.59 \pm 0.11$ & 0.573 & 0.615 \\
\hline
\end{tabular}

Notes: $f: f$ stands for the average surface ECG $f$ amplitude. $f^{\prime}$ : when the $f$ wave reached the maximum amplitude during PICC insertion, it was recorded as f'.

Abbreviations: ECG, electrocardiogram; N, number; PICC, peripherally inserted central catheter.

\section{Accuracy of IC-ECG using X-ray as a gold standard}

There was no statistical difference between X-ray PICC tip position verification and IC-ECG PICC tip position verification among patients with $\operatorname{AF}\left(\chi^{2}=1.31, P=0.232\right)$. If the average $f$ wave amplitude increased $\geq 0.5 \mathrm{~cm}$ compared that of the surface ECG $f$ wave amplitude, IC-ECG was recorded having positive change. If $\mathrm{X}$-ray showed the tip position of PICC was in the lower $1 / 3$ of SVC, X-ray as the gold standard was recorded positive. Utilizing the cutoff point of $\mathrm{f}$ wave change $\geq 0.5 \mathrm{~cm}$, a sensitivity of 0.94 , a specificity of 0.71 , a positive predictive value of 0.98 , and a negative predictive value of 0.42 were observed. Table 3 shows the detailed data.

A receiver operating characteristic curve was calculated to verify the relationship between X-ray PICC tip position verification and IC-ECG tip position verification (Figure 2). The area under the curve was 0.909 (95\% CI: $0.810-1.000$ ), indicating that the accuracy of the IC-ECG PICC tip verification was good. 
Table 3 Accuracy of IC-ECG for tip verification using X-ray as a gold standard

\begin{tabular}{llll}
\hline IC-ECG & X-ray & & Total \\
\cline { 2 - 3 } & Positive & Negative & \\
\hline Positive & 106 & 0 & 106 \\
Negative & 7 & 5 & 12 \\
Total & 113 & 5 & 118 \\
\hline
\end{tabular}

Notes: Positive: if the tip of PICC was in the lower $1 / 3$ of SVC, it was recorded as positive. Negative: if the tip of PICC was not in the lower $1 / 3$ of SVC, it was recorded as negative.

Abbreviations: IC-ECG, intracavitary electrocardiogram; PICC, peripherally inserted central catheter; SVC, superior vena cava.

\section{Discussion}

Since the first report of Hellerstein et al's ${ }^{8}$ study in 1949 regarding CVC placement guided by ECG, numerous studies have been performed to evaluate whether this technique was reliable when placing a CVC. But there have been few studies done on using ECG technology specifically for PICC position verification. ${ }^{5}$ Smith et al's ${ }^{1}$ study found that the peak $P$ wave amplitude was highest at the superior vena cava-right atrial (SVC-RA) junction by performing venography while placing the PICCs under ECG control at $1 \mathrm{~cm}$ intervals. They concluded that with PICC insertion into the right atrial, $\mathrm{P}$ wave amplitude decreased and eventually became negative. ${ }^{1}$ In Pittiruti et al's $\mathrm{s}^{13,14}$ studies, they found that there were no complications potentially related to the method itself, and they drew the conclusion that the intracavitary

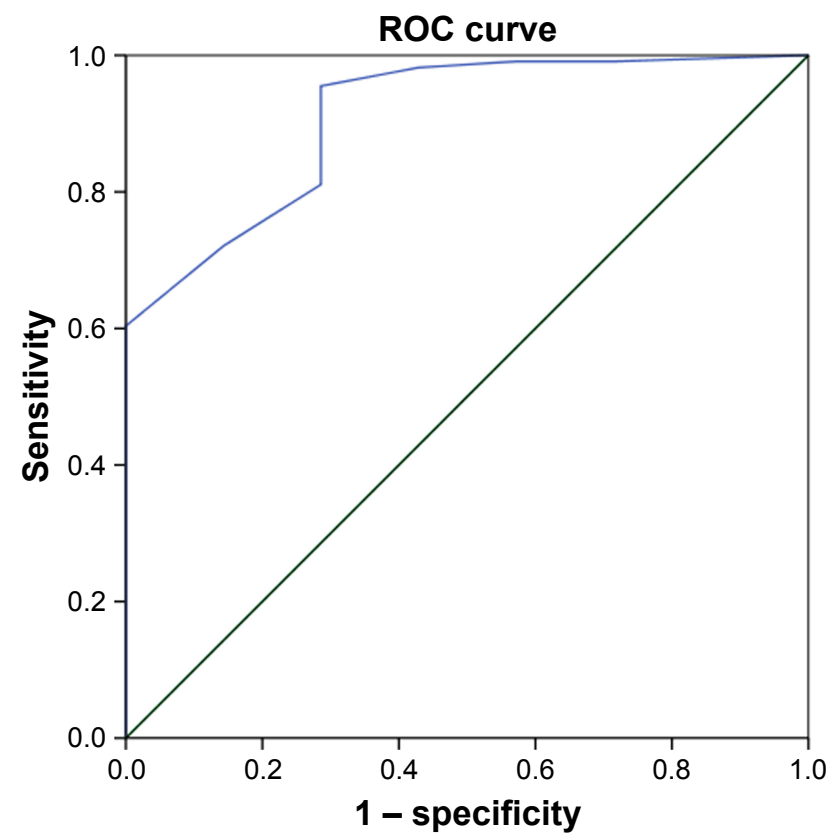

Figure 2 Receiver operating characteristic curve for the relationship between $X$-ray PICC tip position verification and IC-ECG tip position verification.

Abbreviations: IC-ECG, intracavitary electrocardiogram; PICC, peripherally inserted central catheter; ROC, receiver operating characteristic.
ECG method for real-time confirmation of tip position was accurate, safe, feasible in all adult patients, and applicable to any type of short-term or long-term central venous access device. Oliver et al's $s^{9} 5$-year observation found that using ECG to confirm PICC tip position was cheaper, quicker, and more accurate than postprocedural chest X-ray. Zhou et al's ${ }^{15,16}$ studies of the ECG-guided PICC tip placement among neonates found no significant complications occurred in the studied neonates. Their studies suggest ECG-guided tip placement is a promising approach for improving the success rate of tip location when placing a PICC in neonates and the accuracy in the lower extremity was higher than that in the upper extremity among neonates. ${ }^{15,16}$ Rossetti's study suggests that the IC-ECG method is safe and accurate in the pediatric patients. ${ }^{17}$ The studies so far have shown that the ECG-guided method has clear advantages in terms of accuracy and feasibility, does not expose the patient to any radiation, is cheap to run, and provides instant, real-time confirmation of tip position. Furthermore, there is no time delay in commencing the patient on IV therapy. ${ }^{9}, 13,17,18$

However, all the studies above were based on the normal surface ECG because ECG technique relied on interpretation of changes to a patient's $\mathrm{P}$ wave, and patients with rhythms were often in the exclusion criteria from published studies. ${ }^{19,20}$ Besides Engelhardt et al's $\mathrm{s}^{21}$ study on comparing CVC location control by ECG and by chest X-ray in 40 patients with absolute arrhythmia and AF, there were no other data on patients with abnormal surface ECG such as patients with AF with $\mathrm{f}$ wave instead of $\mathrm{P}$ wave. To the best of our knowledge, this study is the first study to evaluate the safety and accuracy of ECG-guided PICC tip position verification applied in patients with AF. In this study, we found that the f wave amplitude changed as the PICC tip went deeper at SVC. When the catheter entered SVC, f wave got higher, which was similar to $P$ wave change of the normal surface ECG patients. When the catheter entered the lower $1 / 3$ of SVC, the amplitude of $f$ wave reached the maximum. When the catheter entered the right atrium, the amplitude of the $f$ wave began to fall. In the normal surface ECG, changes of the P wave morphology are used to track the PICC tip as the tip goes along the SVC to the right atrium. As the PICC tip approaches the sinoatrial node at the CAJ, P wave starts to elevate and reaches the maximum height at the CAJ. When it passes through into the RA, the P wave starts to invert, indicating the PICC is inserted too deep. The ideal end position for the PICC is where the ECG shows maximal P. ${ }^{5}$ Patients with AF have no $\mathrm{P}$ wave, instead it is by $\mathrm{f}$ wave with frequency of 350-600 times/min. The cardiac electrical principles of 
changes of $\mathrm{f}$ wave during the insertion of PICC observed in this study are not clear at present. When the PICC tip goes close to the right atrium, it can stimulate cardiac myocytes to generate potentials. The $\mathrm{f}$ wave is modified by the near field potential and the far field potential. The amplitude of $f$ wave arises along with the deepening of the catheter tip. When the tip of PICC comes to the ideal position (the lower $1 / 3$ of SVC), f wave amplitude is the highest, which is similar to the change of $\mathrm{P}$ wave in the normal surface ECG patients. The mechanism may be that when the catheter tip approaches the right atrium, it stimulates the myocardium to generate electric potential. Under the effect of the near field potential and the far field potential, f wave changes. The amplitude of the f wave increases gradually as the tip of the catheter goes deeper. When the tip of the catheter reaches the ideal position, the amplitude of the f wave also reaches the highest, showing a change similar to the $\mathrm{P}$ wave. ${ }^{21}$ However, further study is required to clarify the detailed f wave change regulation and its specific mechanism.

Regarding the safety of the ECG-guided method used among patients with AF, our study (118 AF patients with 118 PICCs) did not detect any complication related to the use of an intracavitary electrode. The reported incidence of heart rhythm disturbance by ECG method was very low $(0.7 \%) .{ }^{13}$ The operator can know at each moment the ECG waveform, so there is no danger of going too deep. Regarding the accuracy, our study found that there was no statistical difference between X-ray method and IC-ECG method among patients with AF. Using the cutoff point of $f$ wave change $\geq 0.5 \mathrm{~cm}$, a sensitivity of 0.94 , a specificity of 0.71 , a positive predictive value of 0.98 , and a negative predictive value of 0.42 were observed. The area of the receiver operating characteristic curve was 0.909 (95\% CI: 0.810-1.000), indicating that there was high accuracy with the ECG method when X-ray was considered as gold standard. The change of $f$ wave was quantized in our study, which provided relatively objective data for the judgment of $f$ wave. As shown in Table 2, the f wave amplitude increased by $0.59 \pm 0.11 \mathrm{~cm}$ (95\% CI: $0.573-0.615$ ) on average when PICC tip came to the lower $1 / 3$ of SVC.

\section{Limitations}

This study has several limitations. First, the number of samples is relatively less. In clinical application, the f wave morphology can change by the influence of various factors, such as disease and cardiac function, and so the reliability and practicability of ECG method for patients with AF need to be further explored and verified. Second, we only detected the f amplitude changes during PICC placement, and detailed $\mathrm{f}$ waveform changes need to be recorded in a further study. Third, this is only a preliminary observation study, and a random control design will be needed to compare the effectiveness between ECG-guided PICC tip confirmation and X-ray.

\section{Conclusion}

Recent national guidelines (National Institute for Health and Care Excellence, 2015) have advocated the ECG method as a valid alternative to radiological verification of $\mathrm{CVC}$ tip placement. ${ }^{22}$ This study suggests ECG guidance is a safe and effective way of correctly confirming PICC tip position for patients with $\mathrm{AF}$ and can potentially remove the requirement for postprocedural chest X-ray among the patients with AF. Further studies are required to evaluate the detailed fwaveform changes and to compare the effectiveness between ECG-guided PICC tip confirmation and X-ray.

\section{Acknowledgment}

This research was funded and supported by a medical science and technology development project for Shandong Province (2009 HZ024).

\section{Disclosure}

The authors report no conflicts of interest in this work.

\section{References}

1. Smith B, Neuharth RM, Hendrix MA, McDonnall D, Michaels AD. Intravenous electrocardiographic guidance for placement of peripherally inserted central catheters. J Electrocardiol. 2010;43(3):274-278.

2. Dale M, Higgins A, Carolan-Rees G. Sherlock $3 C G(\mathbb{R})$ tip confirmation system for placement of peripherally inserted central catheters: a NICE medical technology Guidance. Appl Health Econ Health Policy. 2016;14(1):41-49.

3. Svendsen MC, Birrer D, Jansen B, et al. Accurate nonfluoroscopic guidance and tip location of peripherally inserted central catheters using a conductance guidewire system. J Vasc Surg Venous Lymphat Disord. 2013;1(2):202-208.e1.

4. Hostetter R, Nakasawa N, Tompkins K, Hill B. Precision in central venous catheter tip placement: a review of the literature. $J$ Assoc Vasc Access. 2010;15(3):112-125.

5. Oliver G, Jones M. ECG or X-ray as the "gold standard" for establishing PICC-tip location? Br J Nurs. 2014;23(Suppl 19):S10-S16.

6. Walker G, Chan RJ, Alexandrou E, Webster J, Rickard C. Effectiveness of electrocardiographic guidance in CVAD tip placement. Br J Nurs. 2015;24(14):S4, S6, S8-S12.

7. Timsit JF, Bouadma L, Ruckly S, et al. Dressing disruption is a major risk factor for catheter-related infections. Crit Care Med. 2012; 40(6):1707-1714.

8. Hellerstein HK, Pritchard WH, Lewis RL. Recording of intracavity potentials through a single lumen, saline filled cardiac catheter. Proc Soc Exp Biol Med. 1949;71(1):58-60.

9. Oliver G, Jones M. ECG-based PICC tip verification system: an evaluation 5 years on. Br J Nurs. 2016;25(19):S4-S10. 
10. Gao Y, Liu Y, Ma X, Wei L, Chen W, Song L. The incidence and risk factors of peripherally inserted central catheter-related infection among cancer patients. Ther Clin Risk Manag. 2015;11:863-871.

11. Alexander M. Infusion Nursing Standards of Practice. Vol 34. San Francisco, CA: Untreed Reads Publishing; 2011.

12. Mahlon MA, Yoon HC. CT angiography of the superior vena cava: normative values and implications for central venous catheter position. J Vasc Interv Radiol. 2007;18(9):1106-1110.

13. Pittiruti M, Bertollo D, Briglia E, et al. The intracavitary ECG method for positioning the tip of central venous catheters: results of an Italian multicenter study. J Vasc Access. 2012;13(3):357-365.

14. Pittiruti M, La Greca A, Scoppettuolo G. The electrocardiographic method for positioning the tip of central venous catheters. J Vasc Access. 2011;12(4):280-291.

15. Zhou L, Xu H, Liang J, Xu M, Yu J. Effectiveness of intracavitary electrocardiogram guidance in peripherally inserted central catheter tip placement in neonates. J Perinat Neonatal Nurs. 2017;31(4):326-331.

16. Zhou LJ, Xua HZ, Xu MF, Hu Y, Lou XF. An accuracy study of the intracavitary electrocardiogram (IC-ECG) guided peripherally inserted central catheter tip placement among neonates. Open Med (Wars). 2017;12:125-130.
17. Rossetti F, Pittiruti M, Lamperti M, Graziano U, Celentano D, Capozzoli G The intracavitary ECG method for positioning the tip of central venous access devices in pediatric patients: results of an Italian multicenter study. J Vasc Access. 2015;16(2):137-143.

18. Elli S, Cannizzo L, Marini A, et al. Posizionamento della punta del catetere durante l'impianto di PICC: analisi retrospettiva dell'affidabilità del metodo ECG vs. controllo radiologico. [Evaluation of tip location reliability of intraprocedural ECG vs. chest Xrays in PICC placement]. Assist Inferm Ric. 2016;35(4):174-179. Italian.

19. Pittiruti M, Scoppettuolo G, La Greca A, et al. The EKG method for positioning the tip of PICCs: results from two preliminary studies. J Assoc Vasc Access. 2008;13(4):179-186.

20. Gebhard RE, Szmuk P, Pivalizza EG, Melnikov V, Vogt C, Warters RD. The accuracy of electrocardiogram-controlled central line placement. Anesth Analg. 2007;104(1):65-70.

21. Engelhardt W, Sold M, Helzel MV. EKG-kontrollierte Plazierung zentralvenöser Katheter bei Patienten mit Vorhofflimmern. [ECGcontrolled placement of central venous catheters in patients with atrial fibrillation]. Anaesthesist. 1989;38(9):476-479. German.

22. Gray M. National Institute for Health and Care Excellence. Eur Heart J. 2015;36(4):195.
Therapeutics and Clinical Risk Management

\section{Publish your work in this journal}

Therapeutics and Clinical Risk Management is an international, peerreviewed journal of clinical therapeutics and risk management, focusing on concise rapid reporting of clinical studies in all therapeutic areas, outcomes, safety, and programs for the effective, safe, and sustained use of medicines. This journal is indexed on PubMed Central, CAS,

\section{Dovepress}

EMBase, Scopus and the Elsevier Bibliographic databases. The manuscript management system is completely online and includes a very quick and fair peer-review system, which is all easy to use. Visit http://www.dovepress.com/testimonials.php to read real quotes from published authors.

Submit your manuscript here: http://www.dovepress.com/therapeutics-and-clinical-risk-management-journal 